\title{
X5Learn: A Personalised Learning Companion at the Intersection of $\mathrm{Al}$ and $\mathrm{HCl}$
}

\author{
María Pérez-Ortiz, Claire Dormann, Yvonne Rogers, Sahan Bulathwela, \\ Stefan Kreitmayer, Emine Yilmaz, Richard Noss and John Shawe-Taylor \\ \{maria.perez,c.dormann,y.rogers,m.bulathwela,emine.yilmaz,r.noss,j.shawe-taylor\}@ucl.ac.uk \\ Department of Computer Science, University College London, London (UK)
}

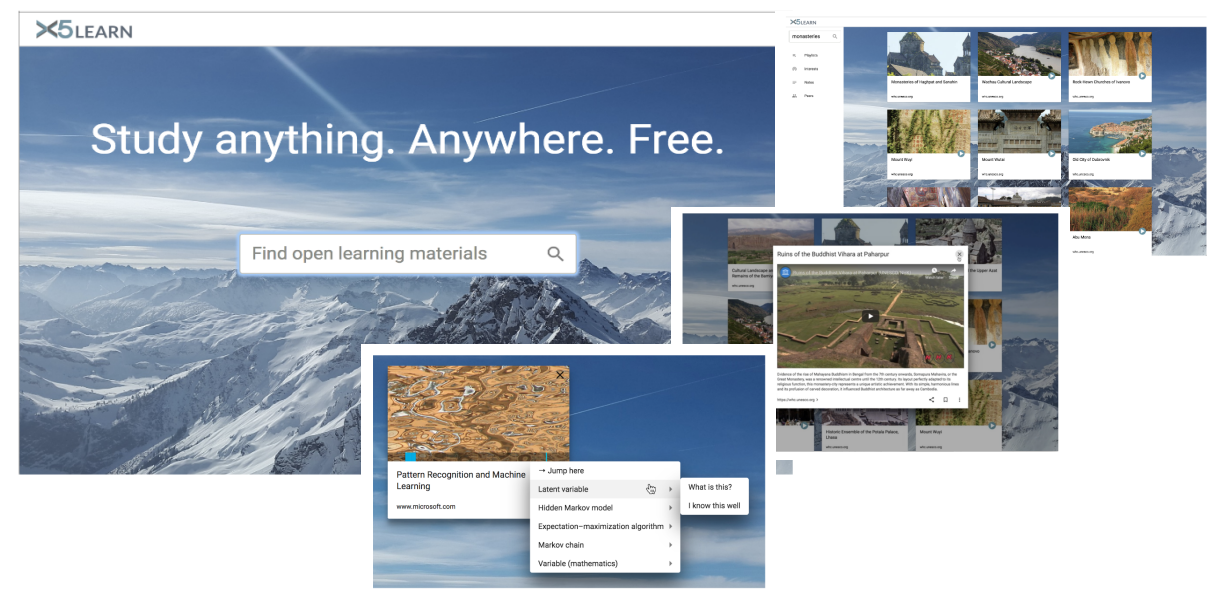

Figure 1: The user interface of X5Learn, showing how a user can search and browse for open educational materials. \begin{abstract}
X5Learn (available at https://x5learn.org) is a human-centered AIpowered platform for supporting access to free online educational resources. X5Learn provides users with a number of educational tools for interacting with open educational videos, and a set of tools adapted to suit the pedagogical preferences of users. It is intended to support both teachers and students, alike. For teachers, it provides a powerful platform to reuse, revise, remix, and redistribute open courseware produced by others. These can be videos, pdfs, exercises and other online material. For students, it provides a scaffolded and informative interface to select content to watch, read, make notes and write reviews, as well as a powerful personalised recommendation system that can optimise learning paths and adjust to the user's learning preferences. What makes X5Learn stand out from other educational platforms, is how it combines human-centered design with AI algorithms and software tools with the goal of making it intuitive and easy to use, as well as making the AI transparent to the user. We present the core search tool of X5Learn, intended to support exploring open educational materials.
\end{abstract}

Permission to make digital or hard copies of part or all of this work for personal or classroom use is granted without fee provided that copies are not made or distributed for profit or commercial advantage and that copies bear this notice and the full citation on the first page. Copyrights for third-party components of this work must be honored. For all other uses, contact the owner/author(s).

IUI '21 Companion, April 14-17, 2021, College Station, TX, USA

(c) 2021 Copyright held by the owner/author(s).

ACM ISBN 978-1-4503-8018-8/21/04.

https://doi.org/10.1145/3397482.3450721

\section{CCS CONCEPTS}

- Information systems $\rightarrow$ Users and interactive retrieval; Personalization; Recommender systems; Search interfaces; • Applied computing $\rightarrow$ Interactive learning environments.

\section{KEYWORDS}

Recommender Systems, Information Retrieval, User Modeling, $\mathrm{Hu}-$ man Computer Interaction, Artificial Intelligence, Intelligent Tutoring Systems, Open Educational Resources, Future User Interfaces

\section{ACM Reference Format:}

María Pérez-Ortiz, Claire Dormann, Yvonne Rogers, Sahan Bulathwela,, Stefan Kreitmayer, Emine Yilmaz, Richard Noss and John Shawe-Taylor. 2021. X5Learn: A Personalised Learning Companion at the Intersection of $\mathrm{AI}$ and HCI . In 26th International Conference on Intelligent User Interfaces (IUI '21 Companion), April 14-17, 2021, College Station, TX, USA. ACM, New York, NY, USA, 5 pages. https://doi.org/10.1145/3397482.3450721

\section{INTRODUCTION}

Artificial Intelligence (AI) in education has great potential for building more personalised curricula, as well as democratising education worldwide. AI in education includes everything from AIdriven, personalized and conversational educative systems, intelligent agents, automatic scoring and assessment and learner-support chatbots, to AI-facilitated matching of learner to learner/teacher and collaborative learning [10]. At the same time, design ideas and principles from Human Computer Interaction (HCI) are beginning to play an increasingly prevalent role. This coming together of fields offers potential for a renaissance of $\mathrm{AI}$ in Education, but 


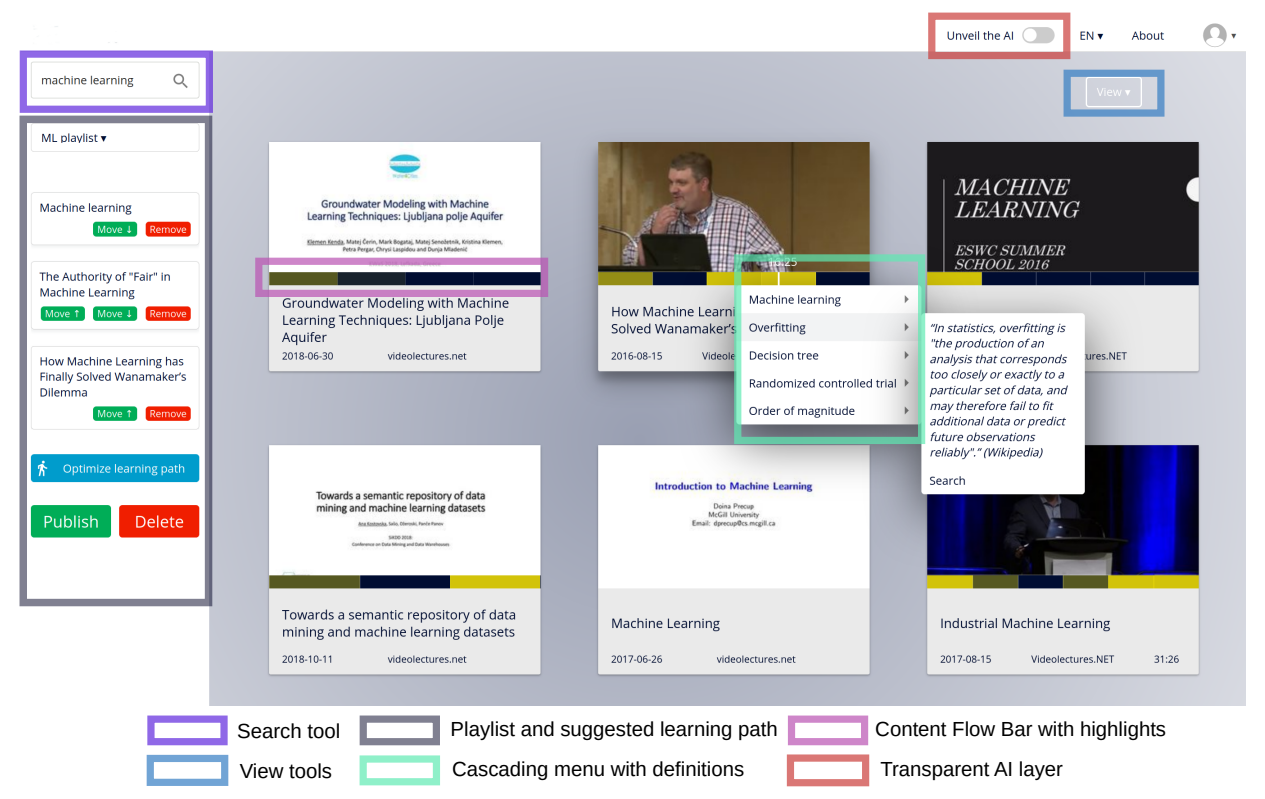

Figure 2: Overview of the platform: The screenshot shows the video player GUI using thumbnails of videos returned by the search engine (with highlighted video clips that cover the searched query), the playlist on the right, the content flow bar for each video clip, the AI function for optimizing the learning path and finally, in the top right corner, the 'unveil the AI' button and the different visualisation functionalities ('view').

with HCI now being central for supporting learners [1, 8, 11, 16], blurring the boundary between search and recommendations and attempting to make learning more interactive, social and personalised [7]. Despite evidence highlighting the benefits of search and navigation tools in education $[1,8,16]$, sophisticated features have yet to become mainstream in educational platforms.

This intersection of $\mathrm{AI}$ and $\mathrm{HCI}$ in education brings to light fundamental research questions (both technical and pedagogical) including what is the nature of knowledge and how can it be understood and represented using AI at the user interface? How best to present this to learners to help them in their various learning activities? How to design a user interface that front ends the AI that is inclusive to all and easy to learn, use and navigate? To this end, we need to ask what are appropriate pedagogy theories that can shape learning? And conversely, what are the key design priorities that can offer learners/teachers intellectual and virtual tools to enhance the learning process?

Our research has begun to explore these questions by designing a holistic AI system that provides content analytics and a educative recommender system, together with a user interface that anyone can use to find free learning resources that match their needs. In this paper, we describe how we designed it: a learning tool intended to provide teachers and learners with a personalised learning platform and unified repository for Open Educational Resources. Our goal was to ensure it was accessible and easy to use, and enabled anyone to "study anything, anywhere, free." (see Figure 1). A portfolio of tools were developed to leverage both AI capabilities and UI functionality. Here, we present the core search tool - X5Learn, intended to support users to easily, confidently and quickly explore new learning materials and to be able to create playlists of the ones selected through a combination of $\mathrm{AI}$ and user decision-making.

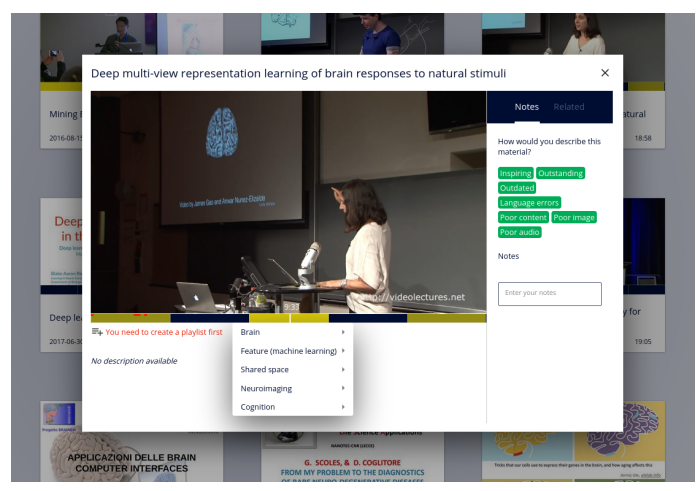

Figure 3: Popup view including the video, the Content Flow Bar and a panel for note-taking and feedback.

\section{THE CORE AI TOOLS AND UI FUNCTIONS}

Open Educational Resources (OERs) are teaching, learning and research materials that are available in the public domain or published under an open license [12]. While there are millions of OERs freely available to all, it is challenging for users to find the right subset of materials. How can we help them discover quickly the best set of OERs rather than spending ages wading through search results that may or may not be suitable? To this end, can we provide a super-powered AI search engine that is front-ended by an aesthetic, easy-to-use and intelligible interface? 
The AI developed for our tool X5Learn was intended to scaffold learning by providing structure and personalisation. It works both in the foreground and background; doing data mining of the videos and suggesting which ones the user might benefit from watching and in what sequence, while enabling the user to take over control at any point and choose for themselves. It works at two levels. First, it transcribes the speech of a video into text and then translates it into English (if it is in another language). Then, it partitions the transcript into fragments of approximately 5 minutes. Each fragment is then annotated using our 'Wikifier' tool which links the fragments with Wikipedia concepts [2]. This approach is domain-agnostic and scalable, avoiding the need for expensive expert labelling resulting in human-interpretable annotations. It is also future-proof as Wikipedia (hence, Wikifier) evolves with the ever changing universal knowledge. The recommender algorithm estimates the quality and difficulty of the video content [4] and builds a model of the skill/knowledge/interest of each user based on previous interactions with the materials [6] and recommends a playlist of materials (e.g. videos) that the algorithm considers will match what is best for the learner's goals and knowledge state.

X5Learn has been designed as a suite of interconnected tools:

- X5GON Connect Service: The Connect Service connects OER repositories around the world into a single network [13], allowing the user to search and navigate all OERs.

- X5GON Translate: This tool automatically generates translations and transcriptions (via automatic speech recognition) in different languages using AI techniques [9].

- X5Learn: This is the core learning platform that presents educational materials returned by our AI search engine and recommendation system (see Figure 2) [3]. The database contains thousands of video lectures. Additionally, books, presentations and scientific papers are also discoverable.

- X5GON Discovery: This is an AI-powered search engine [14] that enables learners to discover learning materials quickly by specifying key parameters (e.g. type of OER, such as video, PDF etc., license available, and preferred language)

- X5GON TrueLearn: This is an AI-based recommendation tool that uses TrueLearn model $[5,6]$ to provide transparent educational recommendations for lifelong learners.

Much thought went into the design of the interface/tools to be interacted by the learner or teacher - so as to give the user both a sense of control and choice. Figure 3 shows some of these features that are listed below and later described in more detail:

- Search Engine: The interface was designed to highlight the part of the materials that best matches a user's search (e.g "find videos about machine learning", see Figure 3). This gives a rationalisation of how the materials are relevant for the query and an efficient cue into the part of the material where the topic relevant content is likely to be.

- Content Flow Bar: This bar creates a narrative of the different topics covered across the videos. It was designed as a UI function to make browsing and searching easier.

- Views: The interface was designed to enable multiple ways of visualising the content of the videos, including thumbnail and visualisations showing presence of keywords throughout a video as illustrated in Figure 5.
- Playlist: The order and priority of selected videos are listed in a window where the AI created OER sequence can be accepted or changed by the user.

- Annotation and review tools: The videos selected can be commented on and reviews added by the user.

- Unveil the AI button: Clicking on the button reveals how and what $\mathrm{AI}$ is being used.

The GUI was designed based on a familiar video player metaphor (see Figure 2). It also integrates a number of well-known visual features, including a search box, popup boxes, cascading menus, timelines and visualisations. One of the novel functions provided in the interface is the Content Flow Bar. After initiating a search, users can then view the results as video thumbnails in a window (showing title and date published). The challenge often faced by the user is that there could be 10,20 or even more videos returned by the search engine and it can be very time consuming for learners to find the most suitable material going through each one sequentially. Below we describe the UX for two of these tools, where it was important to consider how the user would interact and control them: (i) the Content Flow Bar and views and (ii) the Playlist.
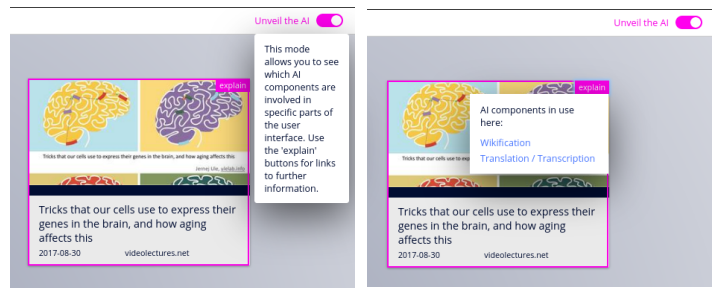

Figure 4: The Unveil the AI explain button, which unveils how and where in the system AI is used.

Content Flow Bar and views. The Content Flow Bar works by providing semantic "snippets" related to the video content that pop up on the screen depicted in the form of a visualisation. The tool enables the user to look rapidly through the content and in doing so be able to see at a glance what is covered in a particular video (and what parts of the video match the search, as highlighted in yellow in figures 2 and 3). As with other video tools (such as YouTube) the user can move their cursor along a timeline and stop it at any point to play from that point. Where it differs from other video players is that it provides keywords that are being referred to in the video in the form of temporally-aware context pop-ups. Moving the cursor from beginning to end will rapidly show what topics are mentioned at any given point. These are extracted using AI tools from the transcript of the video (see Figure 5 and [2]). X5Learn provides additional ways for users to look at the video content. The Content Flow Bar can be expanded to show more information through the use of swimlanes. In each swimlane, different keywords are represented on the timeline by dots of different colours, where each dot is associated with a text snippet extracted from the video content at that time. These additional views enable the user to see more of the temporal flow of the topics in the video in finer granularity. A timeline shows a series of dots that represents one of the topics along with its associated text snippet extracted from the video. Some videos can last as long as 90 minutes. However, the platform 

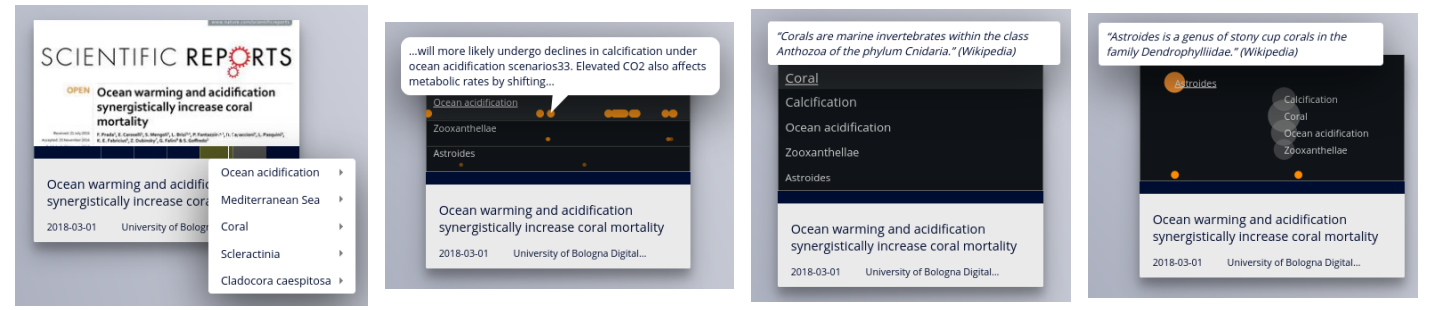

Figure 5: Different views are available to the user. From left to right: thumbnails, swimlanes, topic names and bubbles view. All of the view options include the Content Flow Bar, while the last three also show an ordered list of the most relevant topics.

divides content into smaller pieces. For example, the recommender system works on video clips, rather than whole videos.

The playlist. An additional feature enables users to select clips from videos to create their own playlist. This allows teachers to use X5Learn to prepare a playlist for their courses, while for students it enables them to create a playlist of materials. A note taking feature is also provided that enables users to associate notes to segments of a video. The notes can also be aggregated and downloaded to be shared between students or integrated to coursework. The playlist illustrates how the AI and HCI work together effectively: the AI backbone provides an "optimise learning" function which automatically reorders the video sequences based on its assumed best learning sequence $[5,15]$, while the interface has been designed to enable the user to readily take over control and reorder the video sequence if they are not happy with the recommendations. The system will use interactions with the materials and the system to train the recommender [6].

User studies of X5Learn. A series of usability studies were carried out to determine how understandable and easy to use the new interaction techniques were. The visualisations went through several iterations with groups of 4-6 HCI MSc students taking part as participants using them to carry out specific scenarios. The initial visualisations were found to be overly complicated and difficult to use and so we simplified them to the current set that provided the right level of detail to support navigability.

We have focused on evaluating the tools in the user interface by themselves, first, namely the Content Flow Bar and the Play List. Our future plans are to conduct more in-depth in-the-wild user studies of teachers, students and the general public using the integrated system as a whole. For our initial Content Flow Bar study, we compared user performance and preferences when using the X5Learn video player with Content Flow Bar embedded in it versus a baseline video player. The goal was to understand how the Content Flow Bar supported information seeking, facilitates content navigation and support browsing through video content. 28 participants were asked to find two relevant video clips from a set of 18 educational videos that could be used in teaching a course on (i) Machine Learning and (ii) Climate Change. From these they were then asked to select 4 short and relevant segments for students to watch. A main benefit of this kind of visualisation tool is it enables the user to rapidly look ahead important features - such as how often and where a topic is covered and in doing so helps them to quickly eliminate irrelevant information. The findings showed that participants found browsing the videos using the Content Flow Bar provided an effective look-up tool and a quick summary enabling them to get a better overview of the video. Our results also showed that Content Flow Bar is highly useful in helping users navigate content, with $82 \%$ of participants agreeing that it made finding video clips easier and $93 \%$ thinking that more video players should include similar tools. The interaction logs further showed that this visual tool leads to less time watching content, but more exploration both within and across videos. Participants also navigate deeper and jump (seek) less within the content. Finally, participants take less time before making a relevance judgement. In another user study of the Play List function, we asked a set of teachers to come up with a playlist for the topic of "Artificial Intelligence". Most felt the AI tool was good at coming up with a set of relevant OER videos that were ordered in terms of most relevance. The teachers also liked the option of being able to take over control and reorder the content after the OERs had been selected by X5Learn to match the sequence they wanted their students to look at them in relation to the topics they had chosen for the course outline.

\section{CONCLUSIONS}

We have shown how we can combine AI and HCI to create an integrated suite of tools for teachers and learners. Our initial findings from a series of user studies have demonstrated how this hybrid approach can support fluid interactions; teachers and students happily let the AI make recommendations but then like to take over to finalise the task; by using the novel Content Flow Bar tool to see for themselves what topics are covered in a video. We are also developing a set of methodological approaches that will help us achieve these goals in the short term, while integrating the progress that $\mathrm{AI}$ and $\mathrm{HCI}$ are making in educational contexts. The long-term goal of X5Learn is to build a toolkit of technologies that combines $\mathrm{HCI}$ design principles with AI tools that can improve the experience of searching for and choosing online educational materials.

\section{ACKNOWLEDGEMENTS}

This research is conducted as part of the $\mathrm{X} 5 \mathrm{GON}$ project funded from the EU's Horizon 2020 research programme grant No 761758. This work is also partially supported by the European Commission funded project "Humane AI: Toward AI Systems That Augment and Empower Humans by Understanding Us, our Society and the World Around Us" (grant 820437) and the EPSRC Fellowship titled "Task Based Information Retrieval" (grant EP/P024289/1). 


\section{REFERENCES}

[1] Talea Anderson and Chelsea Leachman. 2019. Strategies for Supporting OER Adoption through Faculty and Instructor Use of a Federated Search Tool. Fournal of Librarianship and Scholarly Communication 7 (2019).

[2] Janez Brank, Gregor Leban, and Marko Grobelnik. 2017. Annotating Documents with Relevant Wikipedia Concepts. In Proc. of Slovenian KDD Conf. on Data Mining and Data Warehouses (SiKDD) (Ljubljana, Slovenia).

[3] Sahan Bulathwela, Stefan Kreitmayer, and María Pérez-Ortiz. 2020. What's in It for Me? Augmenting Recommended Learning Resources with Navigable Annotations. In Proceedings of the 25th International Conference on Intelligent User Interfaces Companion (IUI '20).

[4] Sahan Bulathwela, Maria Perez-Ortiz, Aldo Lipani, Emine Yilmaz, and John Shawe-Taylor. 2020. Predicting Engagement in Video Lectures. In Proc. of Int. Conf. on Educational Data Mining (EDM '20). https://arxiv.org/pdf/2006.00592.pdf

[5] Sahan Bulathwela, Maria Perez-Ortiz, Emine Yilmaz, and John Shawe-Taylor. 2020. Towards an Integrative Educational Recommender for Lifelong Learners. In AAAI Conference on Artificial Intelligence (AAAI '20).

[6] Sahan Bulathwela, Maria Perez-Ortiz, Emine Yilmaz, and John Shawe-Taylor 2020. TrueLearn: A Family of Bayesian Algorithms to Match Lifelong Learners to Open Educational Resources. In AAAI Conference on Artificial Intelligence (AAAI '20).

[7] Ed H. Chi. 2015. Blurring of the Boundary Between Interactive Search and Recommendation. In Proceedings of the 20th International Conference on Intelligent User Interfaces (Atlanta, Georgia, USA) (IUI '15). Association for Computing Machinery, New York, NY, USA, 2.
[8] Renato Cortinovis, Alexander Mikroyannidis, John Domingue, Paul Mulholland, and Robert Farrow. 2019. Supporting the discoverability of open educational resources. Education and Information Technologies 24, 5 (2019), 3129-3161.

[9] Universitat Politècnica de València. 2020. MLLP | Media Transcription and Translation Platform. https://ttp.mllp.upv.es/index.php?page=faq. Accessed: 2020-11-23.

[10] W. Holmes, M. Bialik, and C. Fadel. 2019. Artificial Intelligence in Education: Promises and Implications for Teaching and Learning. Independently Published. https://books.google.co.uk/books?id=tzO7wgEACAAJ

[11] Juho Kim, Philip J. Guo, Carrie J. Cai, Shang-Wen (Daniel) Li, Krzysztof Z. Gajos, and Robert C. Miller. 2014. Data-Driven Interaction Techniques for Improving Navigation of Educational Videos. In Proceedings of the 27th Annual ACM Symposium on User Interface Software and Technology (Honolulu, Hawaii, USA) (UIST '14). Association for Computing Machinery, New York, NY, USA, 563-572. https://doi.org/10.1145/2642918.2647389

[12] UNESCO. 2019. Open Educational Resources (OER). https://en.unesco.org/ themes/building-knowledge-societies/oer. Accessed: 2020-04-01.

[13] X5GON. 2019. X5GON Connect. https://platform.x5gon.org/products/connect. Accessed: 2020-11-23.

[14] X5GON. 2019. X5GON Discovery. https://discovery.x5gon.org/. Accessed: 2020-11-23.

[15] X5GON. 2020. X5GON Learning analytics report. https://www.x5gon.org/wpcontent/uploads/2021/02/D3.2-final.pdf. Accessed: 2021-02-15.

[16] Baoquan Zhao, Shujin Lin, Xin Qi, Ruomei Wang, and Xiaonan Luo. 2018. A Novel Approach to Automatic Detection of Presentation Slides in Educational Videos. Neural Comput. Appl. 29, 5 (March 2018), 1369-1382. https://doi.org/10. 1007/s00521-017-3276-1 